\title{
Growth, Optical and Electrical Properties of zinc tris (thiourea) sulphate (ZTS) Single Crystals
}

\author{
M.Loganayaki \\ Department of Physics, \\ SRM University, Ramapuram, \\ Chennai-600 089
}

\author{
A. Senthil \\ Department of Physics, SRM \\ University, Ramapuram, \\ Chennai-600 089
}

\author{
P. Murugakoothan \\ Postgraduate \& Research \\ Department of Physics, \\ Pachaiyappa's College, \\ Chennai -600030
}

\begin{abstract}
Single crystals of Zinc Tris (thiourea) Sulphate (ZTS) exhibiting high transparency were grown by slow evaporation technique. Single crystal X-ray diffraction analysis reveals that the crystal belongs to orthorhombic system. The optical absorption study reveals the transparency of the crystal and is noticed in the entire visible region and the cut-off wavelength has been found to be $270 \mathrm{~nm}$. The optical band gap was found to be at $3.55 \mathrm{eV}$. The dependence of extinction co-efficient $(K)$ and the refractive index $(n)$ on the wavelength have been observed. The electrical properties of the grown crystal have also been studied using the Cole-Cole plot.
\end{abstract}

\section{Keywords}

Single crystal, slow evaporation technique, Single crystal $\mathrm{X}$-ray diffraction.

\section{INTRODUCTION}

The nonlinear optical properties of the organic molecular crystals have received a great deal of interest for the past two decades due to their extensive application in the fields like laser technology, telecommunication, optical information processing and storage $[1,2]$. The nonlinear response of the crystal is due to the application of strong sources of electric field which gives rise to the second and higher order nonlinear properties like second harmonic generation, d-c rectification, frequency mixing, sum generation and self focusing [3]. One can explore all these nonlinear effects from the asymmetric systems by attributing them into high intense optical beam. The performance of the organic NLO materials in the optical region placed a great demand on these materials owing to which, several attempts have been made by the scientists to identify the good quality nonlinear organic crystals. The search for new frequency conversion materials in recent years has concentrated on semi-organic complexes in which many optically active organic amino acids are mixed with the inorganic salts in order to enhance their physical and chemical properties. The semi-organic materials have the potential for combining the high optical nonlinearity and flexibility of organics with temporal, thermal stability and excellent transmittance of inorganics [4-8]. In this present investigation we report the growth, optical and electrical properties of semiorganic Zinc Tris (thiourea) Sulphate (ZTS) single crystals.

\section{SYNTHESIS}

The starting materials Zinc sulphate heptahydrate and thiourea (AR grade) were dissolved in millipore water $\left(18.2{\mathrm{M} \Omega ~ \mathrm{~cm}^{-1}}^{-1}\right)$ in mole ratio of $1: 3$ at room temperature. The prepared solution was stirred well for 3 hours using a magnetic stirrer to avoid coprecipitation of the material and clear solution was obtained. The solution was taken in a covered container for controlled evaporation and kept at room temperature. After 25 days the ZTS material was crystallized at the bottom of the container. The synthesized material was purified by repeated recrystallization process and used for the growth of crystals. The grown ZTS crystals are shown in figure 1.

\section{RESULTS AND DISCUSSIONS 3.1 Single Crystal X-ray Diffraction Studies}

Single crystal X-ray diffraction analysis for the grown crystal has been carried out to identify the cell parameters using an ENRAF NONIUS CAD 4 automatic X-ray diffractometer with $\mathrm{MoK} \alpha$ radiation $(\lambda=0.71703 \AA)$. It is found that the grown crystal belongs to orthorhombic crystal system with space group $\mathrm{Pca} 2_{1}$ and the lattice parameters are $a=11.126$ $\AA, b=7.773 \AA$ and $c=15.491 \AA$ which agree well with the reported literature [9].

\subsection{Optical Transmission Studies}

The UV-Vis transmission spectrum of ZTS crystal has been recorded in the range $200-1000 \mathrm{~nm}$ by varian $5 \mathrm{E}$ UV-VisNIR spectrophotometer and the spectrum is shown in figure 2 . Low absorbance is observed throughout the visible and IR region. The cutoff wavelength of the ZTS crystal is found to be $270 \mathrm{~nm}$.

The optical absorption co-efficient $(\alpha)$ was calculated using the following relation

$$
\alpha=\frac{1}{d} \log \left(\frac{1}{T}\right)
$$

where ' $T$ ' is the transmittance and ' $d$ ' is the thickness of the crystal.

As a direct band gap material, the crystal under study has absorption co-efficient $(\alpha)$ obeying the following relation for high photon energies $(h v)$ 
$\alpha=\frac{A\left(h v-E_{g}\right)^{1 / 2}}{h v}$

where $E_{g}$ is band gap and $A$ is constant.

The graph between $(\alpha h v)^{2}$ and $(h v)$ is shown in figure 3 and the band gap $\left(E_{g}\right)$ of the crystal was calculated as $3.55 \mathrm{eV}$. As a consequence of wide band gap, the grown crystal has large transmittance in the visible region [10].

\subsection{Determination Of Optical Constants}

The optical constants, refractive index (n) and extinction coefficient $(\mathrm{K})$ were determined from the transmission $(\mathrm{T})$ spectrum. The transmittance of the crystal $[11]$ is

$$
T=\frac{(1-R)^{2} \exp (-\alpha t)}{1-\mathrm{R}^{2} \exp (-2 \alpha t)}
$$

where $t$ is the thickness and $\alpha$ is related to extinction coefficient $\mathrm{K}$ by

$$
K=\frac{\alpha \lambda}{4 \pi}
$$

Figure 4 shows the plot of extinction co-efficient $(K)$ as a function of wavelength. From the graph it is clear that the value of extinction coefficient $(K)$ decreases with the increase of wavelength. The refractive index $(n)$ can be determined from the reflectance $(R)$ using the relation [12].

The Reflectance (R) is written in terms of refractive index (n)

$$
\text { as } \quad R=\frac{(n-1)^{2}}{(n+1)^{2}}
$$

It can also be written in terms of absorption coefficient as

$$
R=\frac{1 \pm \sqrt{1-\exp (-\alpha t+\exp (\alpha t)}}{1+\exp (-\alpha t)}
$$

From the above equation, the refractive index $\mathrm{n}$ can be derived as

$$
n=-\frac{(R+1) \pm \sqrt{3 R^{2}+10 R-3}}{2(R-1)}
$$

wavelength. The internal efficiency also depends on photon energy. Hence by tailoring the photon energy, one can achieve the desired material for optical device fabrications.

\subsection{Electrical Conductivity Studies}

Electrical conduction takes place as a result of electron jump from the low valence state to high valence state as well as movement of ions. The d.c electrical conductivity $\left(\sigma_{\mathrm{dc}}\right)$ of the grown crystal at room temperature is determined using the relation

$$
\sigma_{d c}=\frac{d}{A R_{d c}}
$$

where ' $d$ ' is the thickness of the grown crystal and ' $A$ ' is the area of the grown crystal and $R_{\mathrm{dc}}$ is the d.c resistance of the same crystal. The value of $R_{d c}$ is evaluated from the ColeCole plot drawn between $Z^{\prime}=Z \cos \theta$ and $Z^{\prime \prime}=Z \sin \theta$ and is shown in figure 6 . The d.c conductivity $\left(\sigma_{\mathrm{dc}}\right)$ determined from the Cole-Cole spectra at room temperature is found to be $10.66 \times 10^{-5} \mathrm{mho} \mathrm{m}^{-1}$. The low value of electrical conductivity of the crystal is due to a decrease in the mobility of the charge carriers by ion size, which leads to change in the electronic band structure [13].

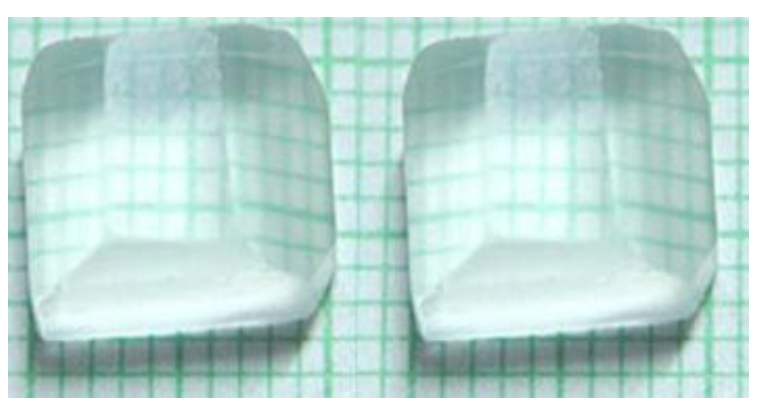

Figure.1 As grown ZTS crystal

It is clear from the figure 5 that refractive index $(n)$ decrease as the wavelength increases. Thus both the values of extinction coefficient $(k)$ and refractive index $(n)$ depend on 


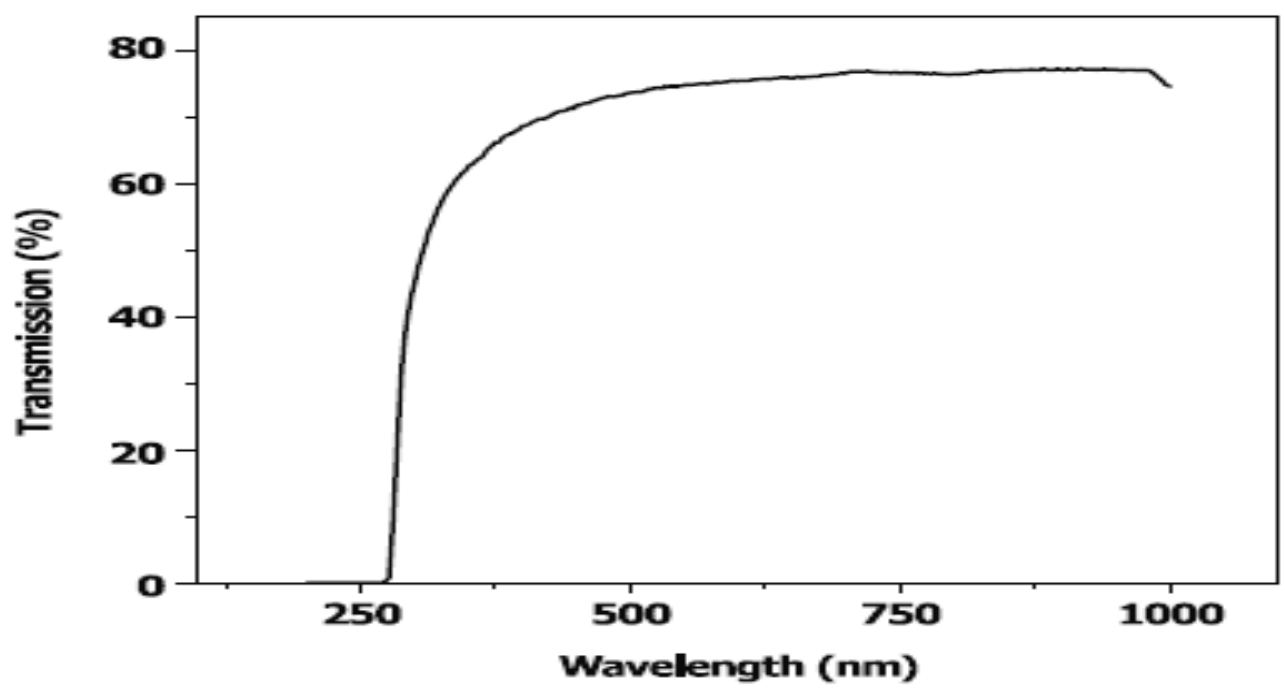

Figure 2. UV-Vis transmission of the ZTS crystal

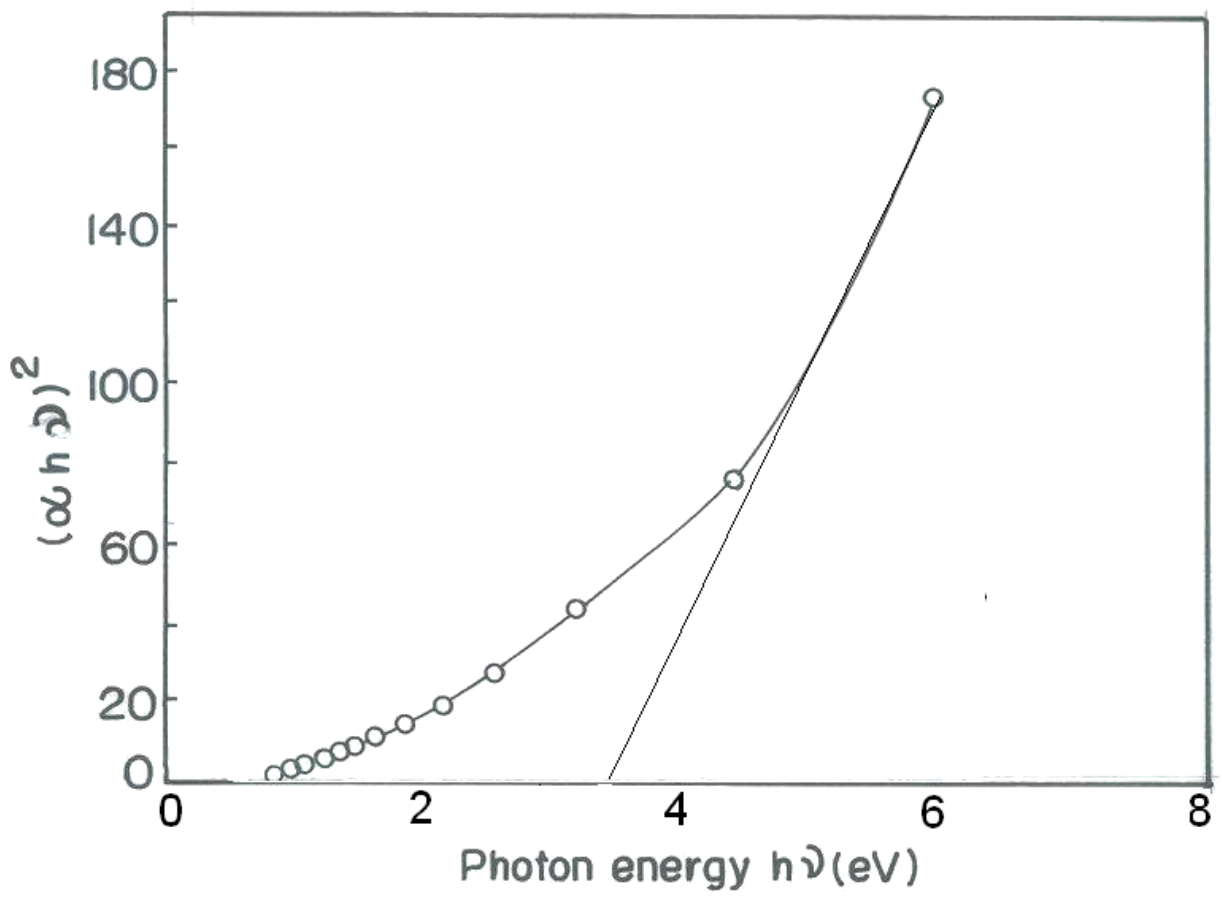

Figure 3. $(\alpha h v)^{2}$ versus $(h v)$ for ZTS crystal. 


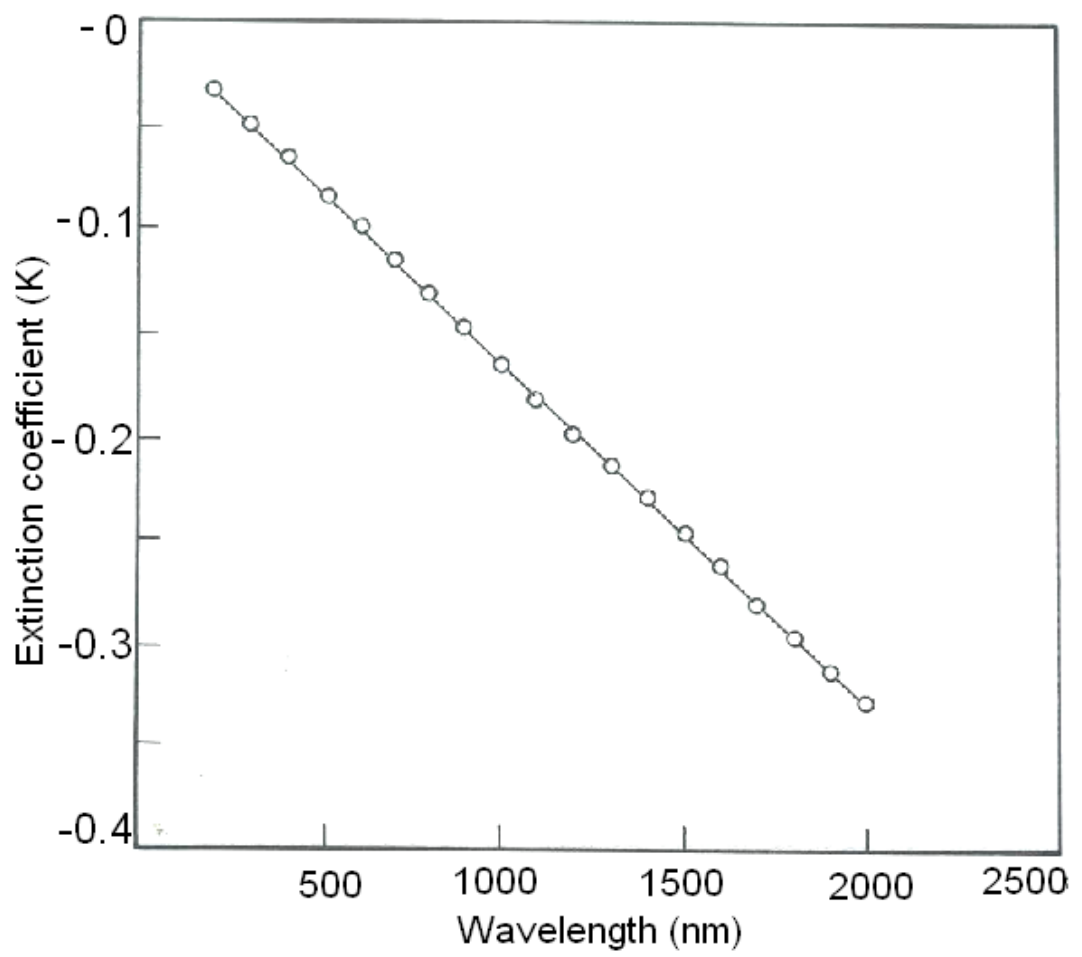

Figure 4. Plot of extinction coefficient $(K)$ versus wavelength

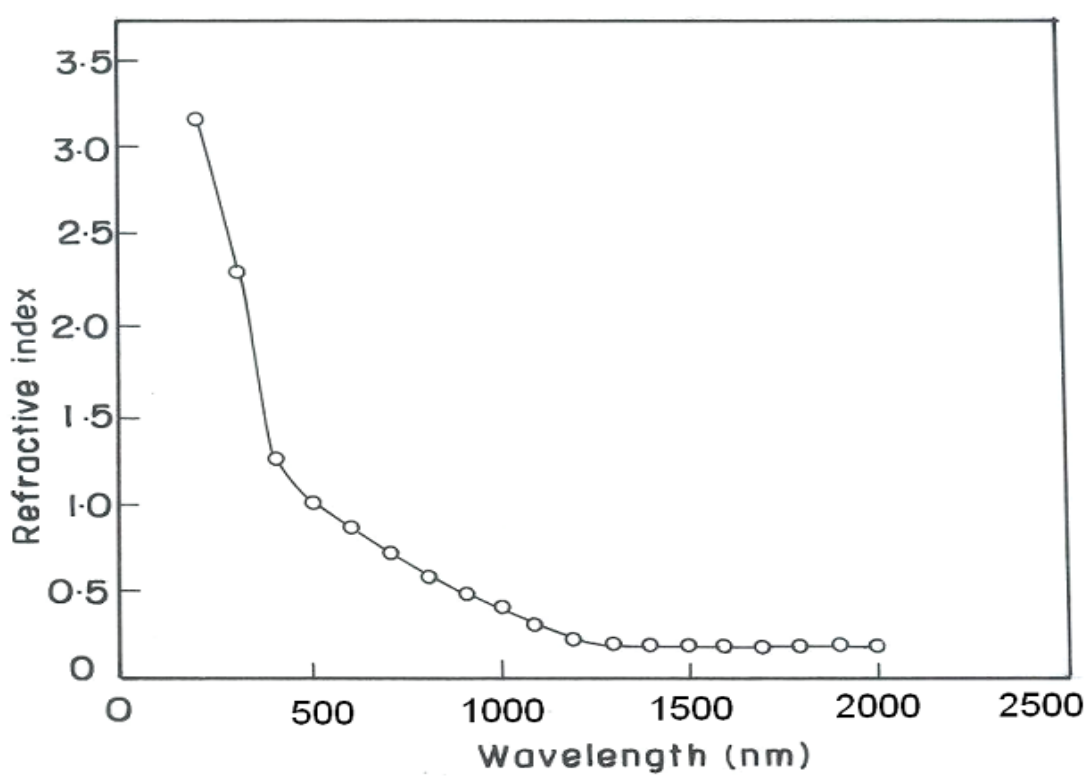

Figure 5. Refractive index $(n)$ versus wavelength 


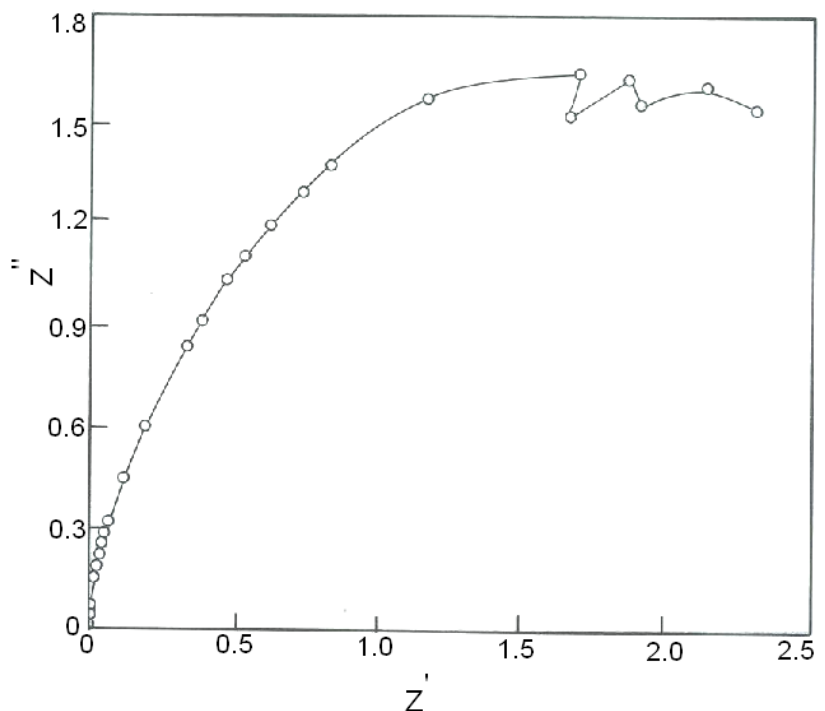

Figure 6. Cole-Cole plot.

\section{CONCLUSION}

Optically good quality single crystals of ZTS were grown by slow evaporation technique within a period of three weeks. The lattice parameters were determined from the single crystal XRD and it has been found that ZTS belongs to orthorhombic crystal system with space group $\mathrm{Pca} 2{ }_{1}$. From UV transmission study, the band gap energy was found to be $3.55 \mathrm{eV}$. The optical investigation such as extinction coefficient $(K)$ and refractive index $(n)$ indicated the transparency of the crystal and confirmed its suitability for optical device fabrication.

\section{REFERENCES}

[1] D. S. Chemla and J. Zyss (Eds.), "Nonlinear Optical Properties of Organic Molecules and Crystals", 1987, Academic Press, NewYork, 1987.

[2] X. Q. Wang, D. Xu, D. R. Yuan, Y. P. Tian, W. T. Yu, S Y. Sun, Z. H. Yang, Q. Fang, M. K. Lu, Y. X. Yan, F. Q.Meng, S. Y. Guo, G. H. Zhang, and M. H. Jiany, 2003, Mater. Res. Bull. 34.1992b.

[3] P. A. Franken and J. F. Ward, Rev. 1963, Mod. Phys. 35, 23

[4] G C Gunjan Purohit Joshi, 2003, Indian J. Pure Appl. Phys. 41,922

[5] P M Ushashree, R Jayavel, C Subramanian and P Ramasamy, 1999, J. Crystal Growth 197, 216.

[6] R Rajasekaran, P M Ushashree, R Jayavel and P Ramasamy, 2000, J. Crystal Growth 218, 365.

[7] H O Marey et al, 1995, Opt. Lett. 20, 252.

[8] N J Long, Angew. 1995, Chem. 34, 21.

[9] G.D. Andreetti, L. Cavalca, A. Musatti, 1968, Acta Cryst. B 24, 683 .

[10] D.D.O. Eya, A.J. Ekpunobi and C.E. Okeke, 2006, Acad Open Internet J. 17.

[11] V. Krishnakumar and R. John Xavier, Spectrochim. 2005, Acta A 60, 709.

[12] A. Ashour, N. El-Kadry and S.A. Mahmoud, 1995, Thin Solid Films 269, 117.

[13] J.H. Lee, S.M. Yoon, B.K. Kim, H.W. Lee and H.S, J. 2002, Mater. Sci. 37, 1165. 